\title{
Effects of weak disorder on stress-wave anisotropy in centered square nonlinear granular crystals
}

\author{
A. Leonard* and C. Daraio ${ }^{\dagger}$ \\ Graduate Aerospace Laboratories (GALCIT), California Institute of Technology, Pasadena, California 91125, USA
}

\author{
A. Awasthi $\mathrm{i}^{\ddagger}$ and P. Geubelle ${ }^{\S}$ \\ Department of Aerospace Engineering, University of Illinois, Urbana, Illinois 61801, USA
}

(Received 23 June 2012; published 21 September 2012)

\begin{abstract}
The present study describes wave propagation characteristics in a weakly disordered two-dimensional granular media composed of a square array of spheres accommodating interstitial cylindrical intruders. Previous investigations, performed experimentally as well as numerically, emphasized that wave-front shapes in similar systems are tunable via choice of material combinations. Here, we investigate the effects of statistical variation in the particle diameters and compare the effects of the resulting disorder in experiments and numerical simulations, finding good agreement.
\end{abstract}

DOI: 10.1103/PhysRevE.86.031305

PACS number(s): 45.70.-n, 46.40.Cd, 05.45.-a

\section{INTRODUCTION}

The nonlinear behavior of highly ordered granular materials, that is, granular cystals, originates from both the Hertzian contact interaction between particles [1] and their inability to transfer tensile loads. Extensive one-dimensional (1D) studies have shown uniform chains of spheres to support the formation and propagation of solitary waves with distinct properties [2-5]. The existence of solitary waves in simple 2D systems has also been observed [6]. Further studies of 2D granular crystals allow for the possible design of complex predetermined loading paths within the system.

Previous studies of the static load transfer path in 2D granular systems showed that ordered granular arrays allow the load to be transmitted primarily along lattice vectors [7-10], while disordered particle arrangements result in complicated force chain networks $[7,10-13]$. The role of force chains in the dynamic load transfer within disordered granular systems has also been studied [14-16]. Unlike disordered systems, the dynamic response of $2 \mathrm{D}$ granular crystals has been shown to possess differing, ordered wave propagation paths based on the underlying particle arrangement [6,17-22]. In earlier studies based on a perfect contact lattice of uniform particles, we showed, using experiments and numerical simulations, the ability to systematically alter the wave-front properties [21,22].

The presence of disorder arising from particle imperfections in real granular arrays is inevitable and results in experimental variability of the load transfer path. A variety of causes could account for the disorder present in real granular assemblies, such as tolerances in the particle size and shape imperfections. Even slight variations in particle sizes can result in the presence of gaps or local compressive forces in the initial contact lattice. It has also been suggested that irregularities in particle surface profiles could lead to deviations from Hertzian behavior and reorganization of particles between successive experiments

\footnotetext{
*andreal@caltech.edu

†daraio@caltech.edu

¥amnaya@illinois.edu

§gubelle@illinois.edu
}

[23]. Other sources of disorder stem from the redundancy in particle contacts in densely packed arrays and the presence of friction $[7,9,10]$.

The particle imperfections, or small defects, act as weak scattering points $[8,24-26]$. While the majority of load is still transferred along the preferential load path, slight misalignments divert some of the force to neighboring particles [8]. For example, several studies have looked at the effects of weak disorder on the static load transfer path of a triangular or hexagonal array of particles subject to a point load [7-10]. The presence of disorder causes the ideal sharply peaked response to broaden; the further away one looks from the loading point, the less pronounced the ideal load path since the disorder compounds [7-10]. Mueggenburg et al. experimentally observed the same trends for both quasistatic and dynamic loading conditions for 3D hep and fcc granular arrays [8].

Several studies considered the effects of weak polydispersity on the dynamic load transfer path in granular crystals [25-29]. However, the studies of 2D crystals [27-29] focused on the effects of disorder on the weakly nonlinear behavior of a hexagonal packing of spheres that is, the case where excitation displacements are small or comparable to static loadings. Coste and Gilles observed that, by increasing the static prestress on a granular array, the initial contact lattice becomes regularized and deviations from Herztian behavior are no longer observed $[27,28]$. In the highly nonlinear regime, previous dynamic studies primarily focused on the effects of relatively large imperfections, such as voids [30,31], significant mass or size deviations [31-34], and cracks [35]. The presence of large defects causes the scattering behavior to become more pronounced, significantly altering the system response from the undamaged case. By further increasing disorder in the form of significant size and material distributions or disordered particle placements, force chain networks arise and we lose the predetermined load transfer path of interest [14-16].

This work is an extension of a previous study [22], in which we showed the ability to methodically alter the shape and properties of the wave fronts traveling through a centered square granular crystal. In this study, we numerically investigate a wider range of particle material properties for 
an ideal crystal. Additionally, this work focuses on the effects of weak polydispersity on the highly nonlinear transient wave propagation in a densely packed granular crystal subjected to a localized impulsive excitation. The incorporation of multiple materials within each test configuration leads to additional complexities in the wave scattering behavior compared to previous studies. We compare experimental results with numerical simulations which have incorporated diameter tolerances of the experimentally tested particles to determine the effects of weak disorder on the wave-front characteristics.

\section{PROBLEM DESCRIPTION AND APPROACH}

The centered square granular crystals studied in this work consisted of a 20-by-20 square packing of spherical particles with central particles (i.e., intruders) residing in the interparticle spaces within the square arrays, effectively forming a smaller 19-by-19 square lattice. To simplify the experimental assembly, cylindrical particles were used as intruders. The cylindrical particles were oriented such that their longitudinal axes were perpendicular to the plane of the $2 \mathrm{D}$ bed. The radii of cylindrical particles [given as $r=(\sqrt{2}-1) R$ ] is fixed by geometry such that they touch the surrounding spheres (radii $R$ ) and their height is chosen to be equal to the diameter of the spherical particles. The initially uncompressed granular crystal is excited impulsively, either centrally or along one edge.

We performed a series of experiments in order to measure the average wave-front characteristics and capture the variability due to imperfections in the initial contact lattices. Numerical simulations were performed for ideal granular crystals as well as for weakly disordered crystals. Here, we use the term ideal granular crystals to refer to assemblies with identical particles placed along even grid spacings. In this study, weakly disordered crystals were modeled by incorporating small random deviations in the particle diameters, resulting in initial contact lattice imperfections.

\section{A. Experimental methods}

Experiments were performed on self-standing granular crystals assembled within a confining box. Custom-fabricated sensor particles, instrumented with miniature triaxial accelerometers, replaced solid spheres at selected locations within the crystal. Refer to [6] for a detailed description of the experimental setup and sensor particles. The acceleration recorded from the sensor particles can be directly compared with the acceleration of the center of mass of each particle obtained from numerical simulations. We performed experiments on four different sphere-cylinder material combinations: (a) steel $^{\mathrm{s}}-\mathrm{ptfe}^{\mathrm{c}}$ (b) delrin ${ }^{\mathrm{s}}-\mathrm{ptfe}^{\mathrm{c}}$, (c) steel ${ }^{\mathrm{s}}-\mathrm{steel}^{\mathrm{c}}$, and (d) delrin ${ }^{\mathrm{s}}$-steel ${ }^{\mathrm{c}}$, where the $\mathrm{s}$ and c superscripts denote sphere and cylinder materials, respectively.

The spherical particles (from [36]) were $19.05 \mathrm{~mm}$ in diameter. The steel spheres had a diameter tolerance of $\pm 0.0127 \mathrm{~mm}(0.0667 \%)$ and the delrin spheres had a diameter tolerance of $\pm 0.0254 \mathrm{~mm}(0.1333 \%)$. The cylinders were machined to fit exactly in the interstitial spaces, with a specified diameter and tolerance of $7.8908 \pm 0.0127 \mathrm{~mm}(0.1609 \%)$.

The system was excited by a striker sphere centrally impacting the granular crystal, in-plane between the 10th and 11 th edge spheres. Since the mass of the impactor can influence the response of the system (causing more than one impact in the case of a heavy striker [37,38]), the striker particles were chosen to be identical to the spherical particles in the granular crystal, either delrin or steel based on the material combination being tested. The striker sphere was given an initial velocity with a solenoid mechanism and the striker velocity was measured just before impacting the system [6]. The average striker velocity was $V_{x}=0.92 \mathrm{~m} / \mathrm{s}$ for the delrin sphere and $V_{x}=0.73 \mathrm{~m} / \mathrm{s}$ for the steel sphere.

In order to capture the wave propagation at variable locations within the system, we collected data for three different sensor configurations (Fig. 1) for each material combination. To capture the variability between successive experiments, we repeated each experiment 15 times before modifying the sensor configurations. Additionally, each material configuration was unpacked, reassembled, and retested

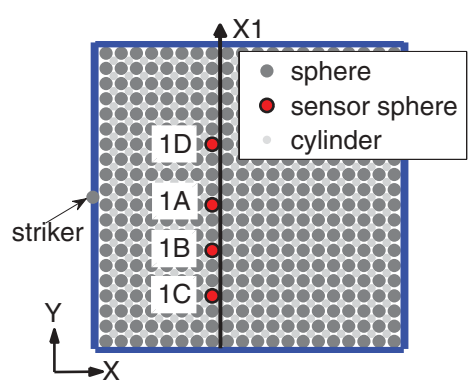

(a)

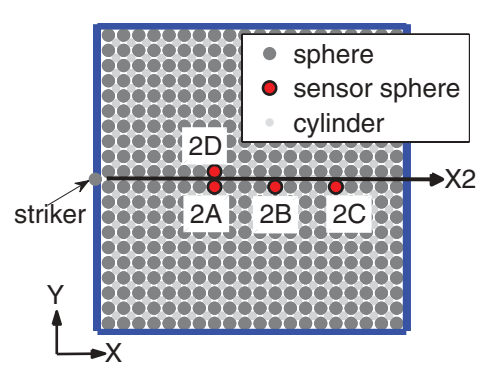

(b)

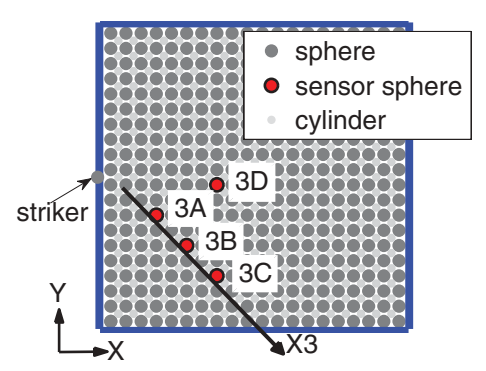

(c)

FIG. 1. (Color online) Schematic diagrams showing the sensor locations in experiments. (a) Sensor configuration 1 had sensors $1 \mathrm{~A}$, 1B, 1C, and 1D located at $X_{8} Y_{10}, X_{8} Y_{7}, X_{8} Y_{4}$, and $X_{8} Y_{14}$ of the 20-by-20 sphere array for all material combinations. (b) Sensor configuration 2 had sensors 2A, 2B, 2C, and 2D located at $X_{8} Y_{10}, X_{12} Y_{10}, X_{16} Y_{10}$, and $X_{8} Y_{11}$ of the 20-by-20 sphere array for the steel ${ }^{\mathrm{s}}$ - ptfe ${ }^{\mathrm{c}}$, delrin -ptfe $^{\mathrm{c}}$, and steel ${ }^{\mathrm{s}}$-steel ${ }^{\mathrm{c}}$ crystals. Sensors 2A, 2B, 2C, and 2D were moved to locations $X_{4} Y_{10}, X_{8} Y_{10}, X_{12} Y_{10}$, and $X_{4} Y_{11}$ of the 20-by-20 sphere array ( 4 spheres closer to the impact), due to small signal amplitudes for the delrin ${ }^{\mathrm{s}}$-steel ${ }^{\mathrm{c}}$, crystals at locations far from the striker impact. (c) Sensor configuration 3 had sensors 3A, 3B, 3C, and 3D located at $X_{4} Y_{8}, X_{6} Y_{6}, X_{8} Y_{4}$, and $X_{8} Y_{10}$ of the 20-by-20 sphere array for all material combinations. For all experiments, the striker sphere impacted the system between the 10 th and 11 th edge spheres with initial velocity $V_{x}$. 
three times for sensor configuration 1 (see Fig. 1), totaling 45 experiments (for each sphere-cylinder material combination) for sensor configuration 1. This unpacking and reassembling allowed us to also capture the variability caused by differences in the initial contact lattices of the granular crystals.

\section{B. Numerical simulations}

In the numerical component of the present work, the individual particles are represented as point masses. There are two types of contacts in the system: one consisting of two spherical particles and another between a spherical and cylindrical particle. These contacts are modeled by nonlinear force-displacement laws as described in Ref. [1] for sphericalspherical contact and [39] for spherical-cylindrical contact at a curved surface. The force-displacement contact law between two spherical solids $i$ and $j$ is given as

$$
\begin{aligned}
\mathcal{F}_{i j}^{s s} & =\frac{4}{3} \frac{E_{s i} E_{s j}}{E_{s j}\left(1-v_{s i}^{2}\right)+E_{s i}\left(1-v_{s j}^{2}\right)} \sqrt{\frac{R_{s i} R_{s j}}{R_{s i}+R_{s j}}} \Delta_{i j}^{3 / 2} \\
& =k_{i j}^{s s} \Delta_{i j}^{3 / 2},
\end{aligned}
$$

where $\mathcal{F}_{i j}^{s s}$ is the contact force acting along the line joining the centers of the $i-j$ pair and the $s s$ superscript indicates an interaction between two spheres, $k_{i j}^{s s}$ is the nonlinear stiffness for two spheres in compression, $E_{s i}, E_{s j}, v_{s i}, v_{s j}, R_{s i}$, and $R_{s j}$ respectively represent the Young's moduli, Poisson's ratios, and radii of spheres, and $\Delta_{i j}$ is the relative displacement of the compressed particles. The nonlinear compressive interaction between cylindrical and spherical particles contacting at their curved surfaces is given as

$$
\begin{aligned}
\mathcal{F}_{i j}^{c s} & =\frac{2}{3} \pi E^{*} \sqrt{2 R_{s}} \sqrt{-\frac{1}{e} \frac{d P}{d e}}\left(\frac{1}{K}\right)^{3 / 2} \Delta_{i j}^{3 / 2} \\
& =k_{i j}^{c s} \Delta_{i j}^{3 / 2},
\end{aligned}
$$

where superscript $c s$ indicates a cylinder-sphere interaction, $E^{*}=E_{s} E_{c} /\left[E_{s}\left(1-v_{c}^{2}\right)+E_{c}\left(1-v_{s}^{2}\right)\right], E_{c}$ and $v_{c}$ are, respectively, the Young's modulus and Poisson's ratio of the cylindrical particles, $e$ is the eccentricity of ellipse of contact between spherical and cylindrical particles, and $K$ and $P$ are the complete elliptic integrals of the first and second class, respectively, with elliptic modulus $e$ [39], with $e=\sin \alpha$, where $\alpha$ is the angular eccentricity. For both spherical-spherical and spherical-cylindrical contacts, the force-displacement law only applies when the particle pairs are in compression; the interaction force is set to zero whenever the particles separate. Materials of both spherical as well as cylindrical particles are assumed to be linear elastic and any effects due to material nonlinearity, plasticity, and dissipation are assumed to be absent.

We prescribe boundary and initial loading conditions, and the dynamic evolution of the granular system is evaluated using an explicit time scheme with the granular module [40] of the LAMMPS molecular dynamics (MD) package [41], specially modified to allow for both cylinder-sphere and sphere-sphere contacts. The particle material properties used in the numerical simulations are given in Table I.

Two types of simulations are performed in the present work. The first evaluates the wave propagation response of
TABLE I. Material properties used in numerical simulations [22].

\begin{tabular}{lccc}
\hline \hline & $\begin{array}{c}\text { Density } \\
\left(\mathrm{kg} / \mathrm{m}^{3}\right)\end{array}$ & $\begin{array}{c}\text { Young's } \\
\text { modulus (GPa) }\end{array}$ & $\begin{array}{c}\text { Poisson's } \\
\text { ratio }\end{array}$ \\
\hline Stainless steel (type 316) & 8000 & 193 & 0.30 \\
Delrin & 1400 & 3.1 & 0.35 \\
PTFE & 2200 & 0.5 & 0.46 \\
\hline \hline
\end{tabular}

ideal (nonrandom) granular crystals while the second examines the wave propagation features in weakly disordered granular lattices.

\section{Nonrandom crystals}

Numerical simulations of nonrandom crystals are performed on two setups. The first configuration is an extended pack of the centered square lattice which incorporates approximately 10000 of both spherical particles and interstitial cylindrical intruders. For this extended system, we examined a wide space of cylinder-sphere mass and stiffness ratios in order to observe the different regimes of wave propagation. The extended system is perturbed symmetrically by giving equal initial velocities to four spheres (Fig. 2), and the different regimes of wave propagation are analyzed by monitoring the total compressive force, which for the $i$ th particle is defined as

$$
F_{i}=\sqrt{\sum_{j=1}^{N_{i}}\left[\left(F_{x j}\right)^{2}+\left(F_{y j}\right)^{2}\right]},
$$

where $N_{i}$ denotes the number of particles adjacent to particle $i$ and $F_{x j}$ and $F_{y j}$ are, respectively, the $x$ and $y$ components of contact force due to particle $j$ on particle $i$. Prior numerical studies of wave propagation in a sphere-sphere system demonstrated the existence of different regimes of propagation which directly depended on the mass and stiffness ratios [21]. Here, a parametric study is performed to assess the influence of the mass, $m_{c} / m_{s}$, and stiffness, $E_{c} / E_{s}$, ratios on the nature of propagation in the sphere-cylinder system.

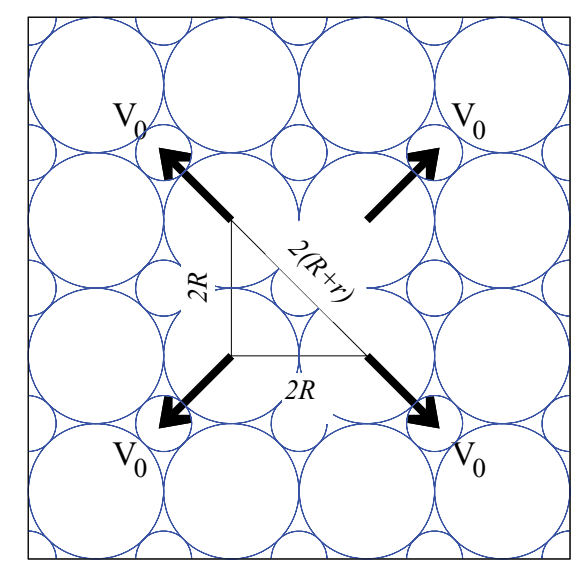

FIG. 2. (Color online) Schematic of extended granular crystal analyzed in the present study. Larger sized granules represent spheres (radius, $R$ ) while smaller ones are cylinders (radius, $r$ ). Wave propagation studies are performed on an extended set of this packing by giving a symmetric disturbance, setting initial velocities on four spherical granules as shown. 
The second setup is a smaller system identical to the to the experimental setup. Numerical simulations of nonrandom crystals corresponding to the experimental configuration were also performed for each of the four sphere-cylinder material combinations tested in experiments (Fig. 1). For these simulations, the impulse excitation is created with a spherical particle impacting the crystal between the 10th and 11th edge spheres, with initial velocity $V_{x}$ (measured from experiments).

\section{Weakly disordered granular crystals}

Numerical simulations of weakly disordered granular crystals were performed for each of the four sphere-cylinder material combinations tested in experiments $\left(\right.$ steel $^{\mathrm{s}}-\mathrm{ptfe}^{\mathrm{c}}$, delrin $^{\mathrm{s}}-\mathrm{ptfe}^{\mathrm{c}}$, steel $^{\mathrm{s}}-$ steel $^{\mathrm{c}}$, and delrin ${ }^{\mathrm{s}}-$ steel $\left.^{\mathrm{c}}\right)$. These numerical studies were conducted over a setup similar to the experimental system described in Fig. 1, and the setup was impacted from the side by a striker sphere. To describe the variation in radii numerically, a normal random distribution was chosen around the mean radius for both types of granules. We assumed tolerance values correspond to 6 standard deviations, which is a common statistical interpretation for mechanical tolerances [42].

To compute the initial configuration for each realization (each initial contact lattice), a set of normally distributed random radii for cylindrical and spherical granules was randomly assigned to the particles of the nonrandom crystal. Mismatching radii placed on perfect lattice sites cause some contacts to separate and others to compress. To achieve an equilibrated starting configuration, the particles were first
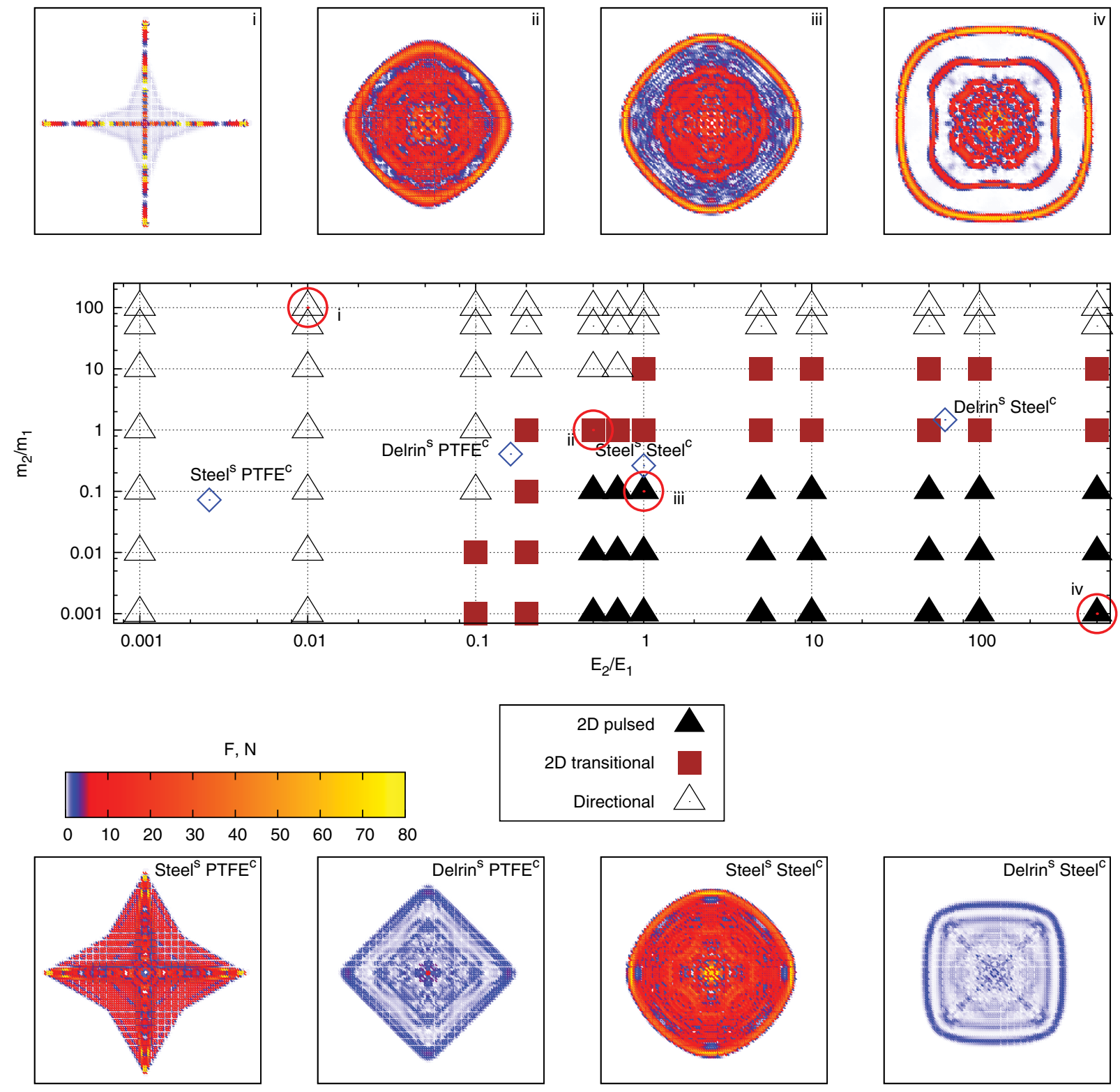

FIG. 3. (Color online) (Center) Different wave propagation regimes mapped on mass $\left(m_{2} / m_{1}\right)$ and stiffness $\left(E_{2} / E_{1}\right)$ ratio space for the cylinder-sphere (2-1) system. (Top) Diagrams depicting examples of different wave fronts achievable in the systems at selected mass and stiffness ratios, indicated with circles in the central figure. (Bottom) Four insets depicting the propagating wave-front shapes for the four material systems tested in experiments, indicated with diamond-shaped symbols in the central figure. 
subjected to a gravitational field to ensure a tightly packed configuration. The random particle velocities arising from the disorder were damped by applying a viscous damping force on each particle, which was proportional to the particle velocity. The strength of the gravitational field and the viscous damping force were gradually reduced until contact forces due to gravity were eliminated and the whole system of particles was consolidated. The settled weakly disordered system was impacted to study the wave propagation.

The effects of disorder were investigated in two ways. The first one consisted of preparing multiple random realizations of the granular crystal and impacting each individual realization once (MR-SI, multiple realizations with single impacts). The second approach involved impacting a single realization multiple times (SR-MI, single realization with multiple impacts). For each study of MR, 15 realizations were prepared. Additionally, for several SR (chosen from the $15 \mathrm{MR}$ ) the initial configuration was impacted 15 times, allowing the system to come to rest between impacts. The acceleration time response of individual particles was monitored and compared with experimental results.

\section{RESULTS AND DISCUSSION}

\section{A. Nonrandom system response}

The present sphere-cylinder granular system supports a variety of propagating wave fronts which are tunable through the choice of mass and elastic moduli (stiffness) ratios as shown in Fig. 3. There are two clearly distinguished wave-front regimes: (1) lattice-directed or directional propagation and (2) dispersed or 2D propagation. Within the dispersed propagation category, we further distinguish two types of transient wave propagation: (2a) pulsed and (2b) transitional. The directional wave propagation is characterized by pulses traveling along the lattice directions [Fig. 3(i)], similar to the case of a square array of spheres without interstitial cylinders [6]. This

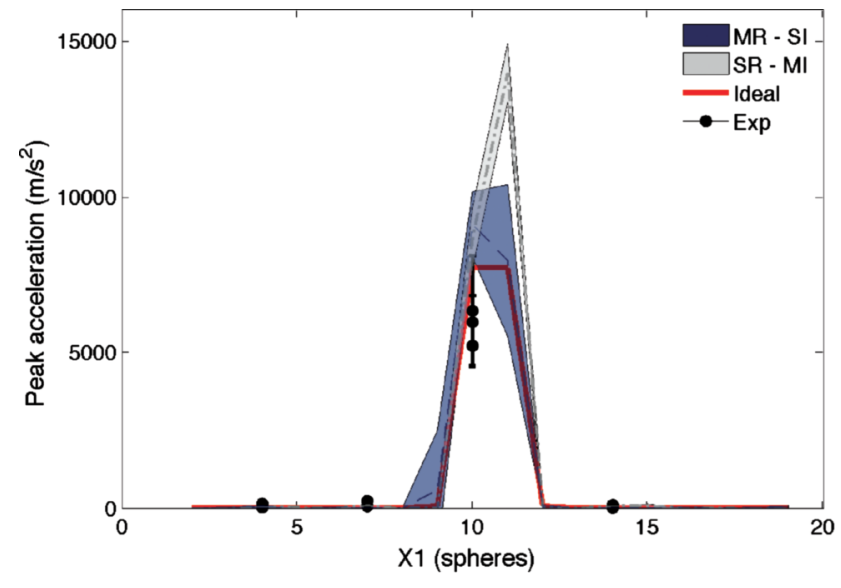

(a)

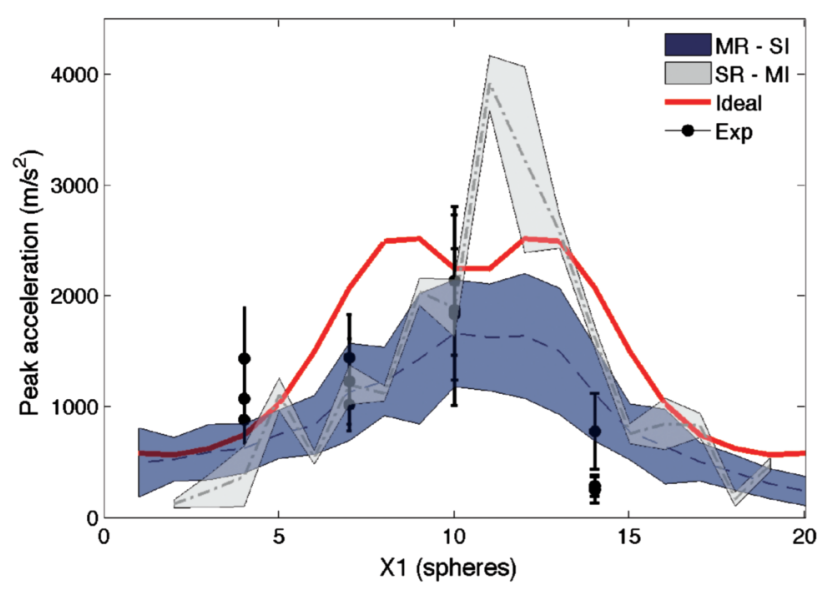

(c)

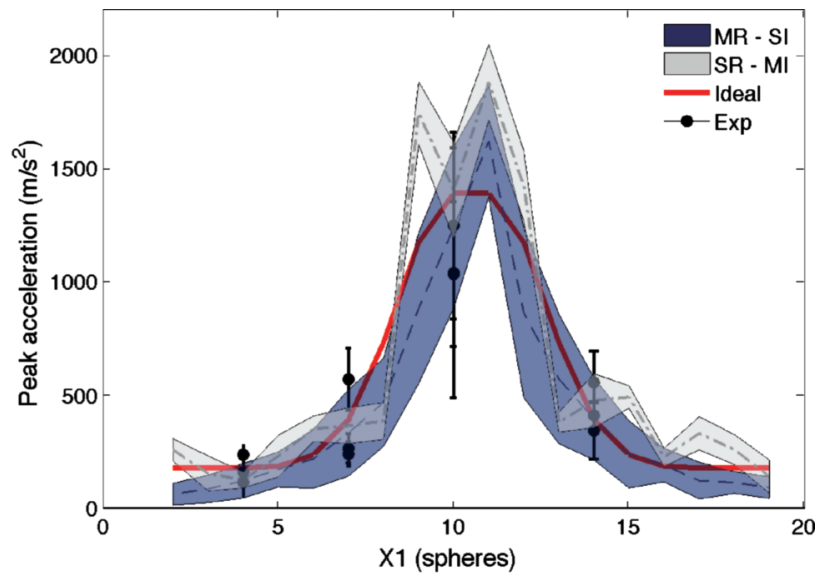

(b)

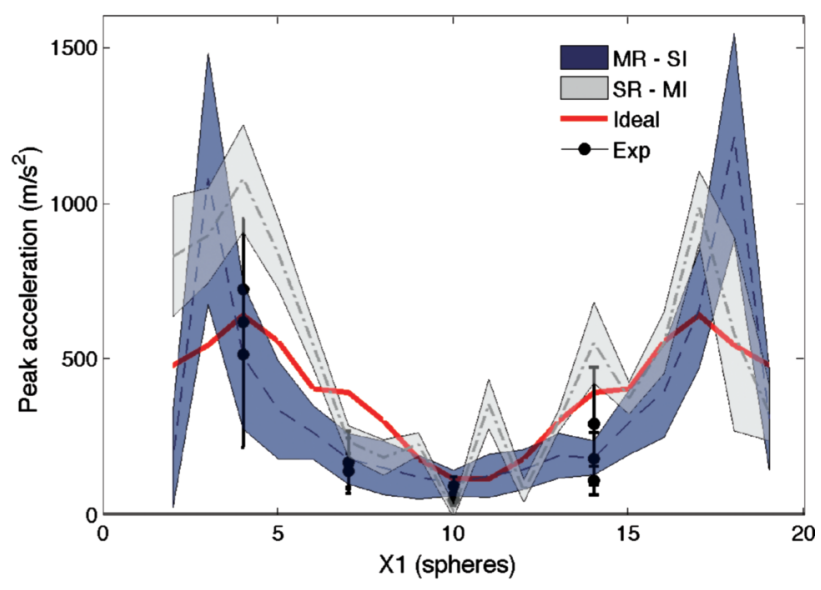

(d)

FIG. 4. (Color online) Peak acceleration $\left(\mathrm{m} / \mathrm{s}^{2}\right)$ in the wave front as a function of $\mathrm{X} 1$ (in units of spheres) for each sphere-cylinder material combination: (a) steel ${ }^{\mathrm{s}}-\mathrm{ptfe}^{\mathrm{c}}$, (b) delrin ${ }^{\mathrm{s}}-\mathrm{ptfe}^{\mathrm{c}}$, (c) steel ${ }^{\mathrm{s}}-\mathrm{steel}^{\mathrm{c}}$, and (d) delrin ${ }^{\mathrm{s}}$-steel ${ }^{\mathrm{c}}$. The results from numerical simulations for the ideal granular crystal are shown by the solid (red) lines. The mean and standard deviation are represented by a dashed lines and dark (blue) shaded regions for the MR-SI (15 realizations each with a single impact) and by dot-dashed lines and light (gray) shaded for the SR-MI (a single realization with 15 impacts).The experimental data are represented by black dots with error bars for the three realizations tested with 15 impacts each. 
directional behavior is characteristic of systems having a low stiffness ratio and high mass ratio. The $2 \mathrm{D}$ pulsed regime is characterized by a convex wave front, with either a square or a circular propagating wave-front shape [Fig. 3(iv)]. This propagation regime occurs for mass ratios less than 0.1 and stiffness ratios greater than 0.5 . Systems with wave fronts in the $2 \mathrm{D}$ transitional regime propagate without the presence of a distinct leading pulse and were observed to support a wide range of wave-front shapes (Figs. 3(ii) and 3(iii)].

The present results are consistent with the findings of previous reports on spherical-spherical systems [21]. The particle configurations tested experimentally are highlighted by diamond-shaped symbols in Fig. 3, and their wave-front shapes in the extended system are shown in the bottom panels of Fig. 3. The very low stiffness ratio inherent in the steel ${ }^{\mathrm{s}}-\mathrm{ptfe}^{\mathrm{c}}$ system causes the wave propagation to be directional. While the other experimental systems $\left(\right.$ delrin $^{\mathrm{s}}-\mathrm{ptfe}^{\mathrm{c}}$, steel $^{\mathrm{s}}$-steel ${ }^{\mathrm{c}}$, and delrin $^{\mathrm{s}}$-steel ${ }^{\mathrm{c}}$ ) all lie in the transition regime, the shape of the propagating front is significantly different for each material combination. As discussed in [22], the trends observed in experiments were found to be in good agreement with numerical simulations on nonrandom granular crystals.

\section{B. Weakly disordered system response}

We now study how the presence of disorder in experiments and numerical simulations affects the response of the system and compare the response with that of ideal granular crystals. We focus our discussion primarily on how the disorder affects the shape of the propagating wave fronts. As discussed in Ref. [22], we can characterize the shape of the wave front by the relative arrival times or by the wave-front amplitudes between sensor locations. However, in our system, these two parameters are not independent. Several studies have confirmed a specific wave-speed-amplitude-scaling relation for 1D systems [3-5]. In 2D systems, we expect a similar behavior, with waves of larger amplitudes traveling faster in specific directions [22].

For the chosen sensor configurations, the shape of each wave front can best be captured by looking at the relative amplitudes and arrival times of the signals along an axis perpendicular to the impact, for example, the X1 direction (see Fig. 1). The peak accelerations in the wave front along the $\mathrm{X} 1$ direction are shown in Fig. 4 for the four material systems under consideration. In the case of the steel $^{\mathrm{s}}-\mathrm{ptfe}^{\mathrm{c}}$ crystal [Fig. 4(a)], the two central rows of spherical particles have a significantly higher amplitude than the remaining particles extending to the crystals edge, clearly signifying the highly directional shape observed in the ideal system (Fig. 3). For the delrin ${ }^{\mathrm{s}}$-ptfe $^{\mathrm{c}}$ crystal [Fig. 4(b)], we see a similar trend, with significantly larger amplitude at the central particles, but with a more gradual decrease in amplitude as the sphere locations approach the edges, corresponding to a triangular wave-front shape. The peak acceleration distribution for the steel $^{\mathrm{s}}$-steel ${ }^{\mathrm{c}}$ crystal [Fig. 4(c)] is smoother, with comparable amplitudes along X1, corresponding to the circular wave front predicted for the ideal system. Finally, for the delrin ${ }^{\mathrm{s}}$-steel $^{\mathrm{c}}$ crystal [Fig. 4(d)], the largest amplitudes are now located close to the crystal edges and the smallest amplitudes at the center of the domain, indicating the squared, redirective wave-front

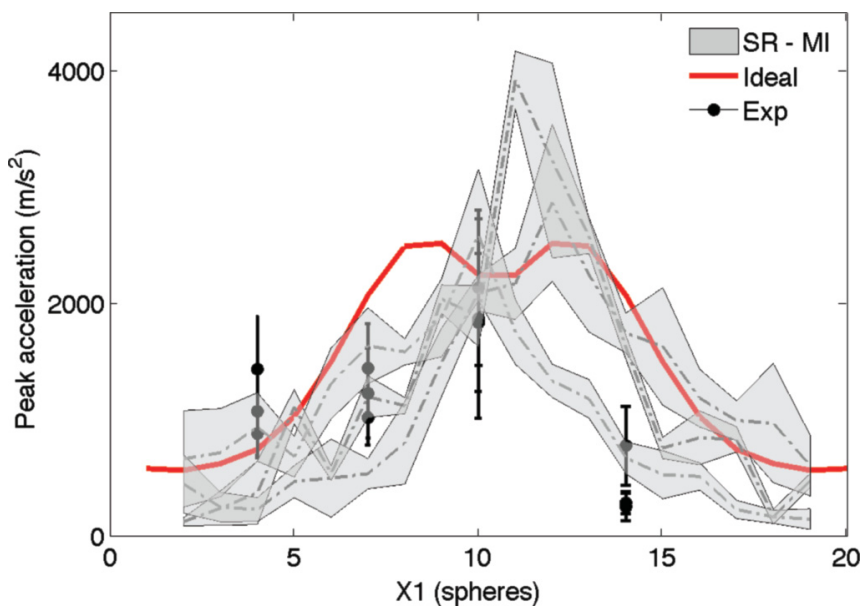

FIG. 5. (Color online) Peak acceleration $\left(\mathrm{m} / \mathrm{s}^{2}\right)$ in the wave front as a function of X1 (in units of spheres) for the steel ${ }^{\mathrm{s}}$-steel $^{\mathrm{c}}$ crystal. The results from numerical simulations for the ideal granular crystal are shown by the solid (red) lines. The mean and standard deviation for three single realizations with 15 multiple impacts (SR-MI) are represented by dot-dashed lines and shaded light gray regions. The experimental data are represented by black dots with error bars for the three realizations tested with 15 impacts each.

shape observed in the ideal crystal numerical simulations (Fig. 3).

The effects of the weak disorder on the wave-front shapes of the four tested material systems are also illustrated in Fig. 4. The wave shapes depicted by average values of peak acceleration along X1 obtained from MR-SI (multiple realizations with single impacts) on each system are consistent

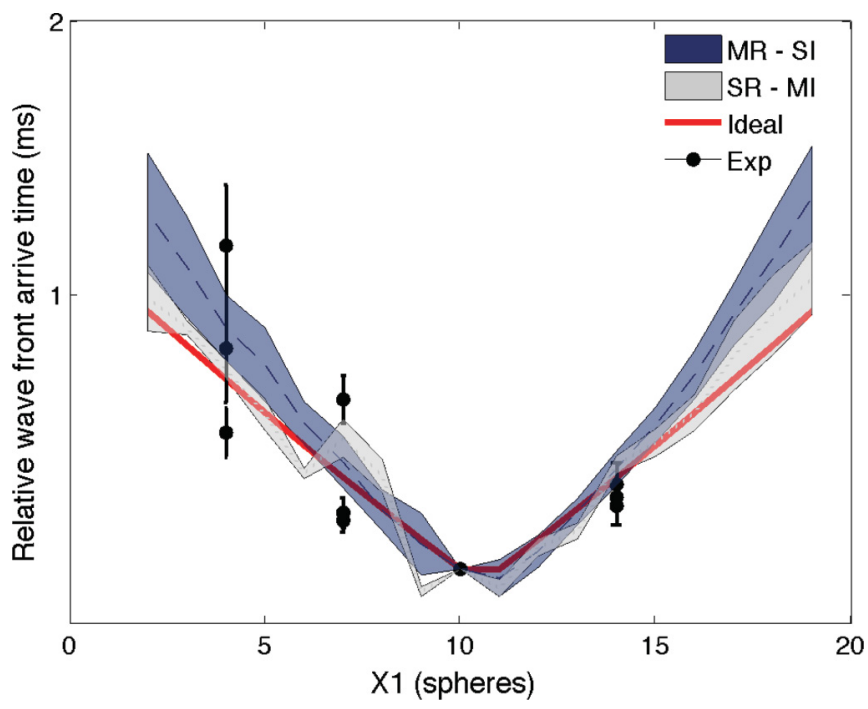

FIG. 6. (Color online) Relative wave-front arrival times along $\mathrm{X} 1$ (spheres) with respect to sensor location 1A, for the delrin ${ }^{\mathrm{s}}-\mathrm{ptfe}^{\mathrm{c}}$ crystal. The results from numerical simulations for the ideal granular crystal are shown by the solid (red) lines. The mean and standard deviation are represented by a dashed line and dark (blue) shaded region for the MR-SI and by a dot-dashed line and light (gray) shaded region for the SR-MI. For each sensor location along X1, three sets of black dots and error bars represent the mean and standard deviation for the three single experimental realizations with multiple impacts. 
with those predicted for the respective ideal crystals. Additionally, the average peak acceleration at sensor locations in the three experimental realizations of each system generally lie within the distributions predicted through MR-SI. Previous 1D studies have shown that the presence of weak disorder results in attenuation of the leading pulse [25,26]. We observe a similar trend in the numerical simulations, in which the average wave-front amplitude predicted through MR of weakly disordered systems is slightly lower than that of the ideal, nonrandom, system.

While the overall trends for individual particle responses from MR closely resembles the ideal system, any single realization (SR) may produce larger deviations from the ideal system response. This is illustrated in Fig. 4, in which a randomly selected SR (one of the MR) is shown for each sphere-cylinder system. The distribution of wave front amplitude for an SR depends on the underlying initial contact lattice and does not vary significantly over multiple impacts (MI), which may cause slight particle rearrangements. The wave-front amplitude distributions along X1 for three SR-MI of the steel ${ }^{\mathrm{s}}-$ steel $^{\mathrm{c}}$ system are shown in Fig. 5. The variability in wave-front amplitude distribution between SR is evident and arises from slight particle misalignments which are unique to the individual contact lattice of an SR.

Another way to characterize the effects of disorder on the wave-front shapes is to consider the relative arrival times between sphere locations along X1 (Fig. 6). Here, we present only the arrival times for the delrin ${ }^{\mathrm{s}}-\mathrm{ptfe}^{\mathrm{c}}$ crystal, since the results show similar information as the peak acceleration plots (Fig. 4). As the spheres are located further from the central particles, the arrival times increase linearly with distance from the impact location, consistent with a triangular wave front. Similar to the distribution of wave amplitudes, we note that the variability, or standard deviation in arrival times, associated with MR is greater than that emerging from MI. Additionally, for both SR and MR, the scatter observed in the results is more pronounced near the sides of the primary wave front. This is expected since the waves must travel through more particle contacts and hence encounter more interfaces than the middle portion. We find good agreement between the numerical results and the signal variability measured experimentally.

Next, we present in Fig. 7 the acceleration-time curves measured by our sensors at each particle location. These local measurements allow for a more direct comparison of the signal
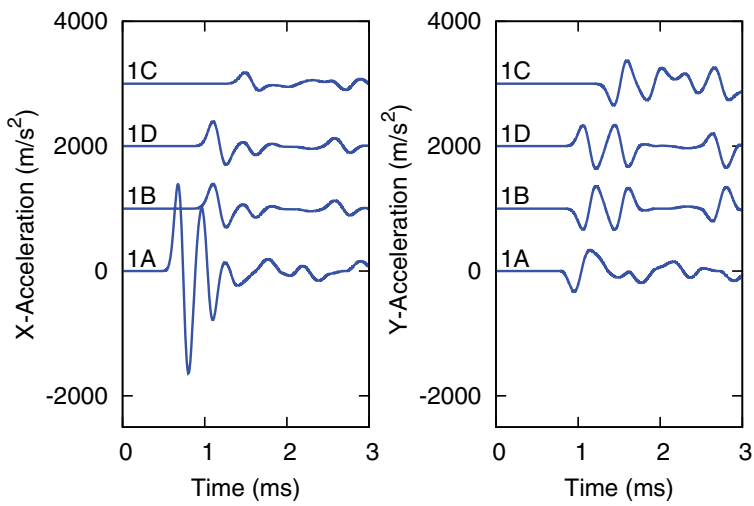

(a)
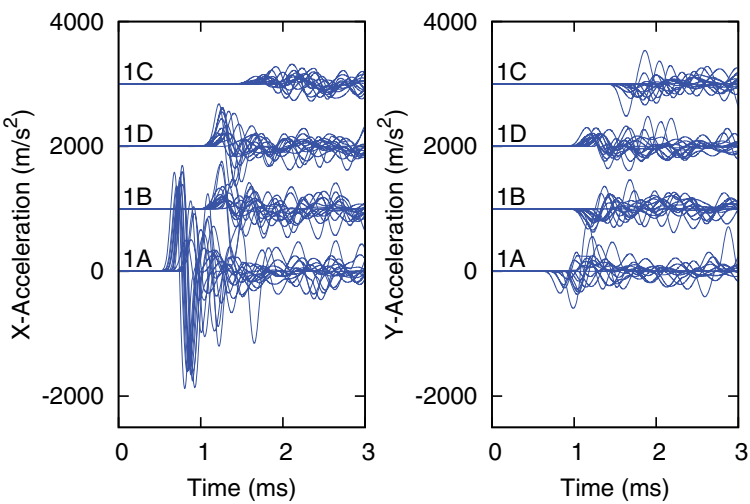

(c)
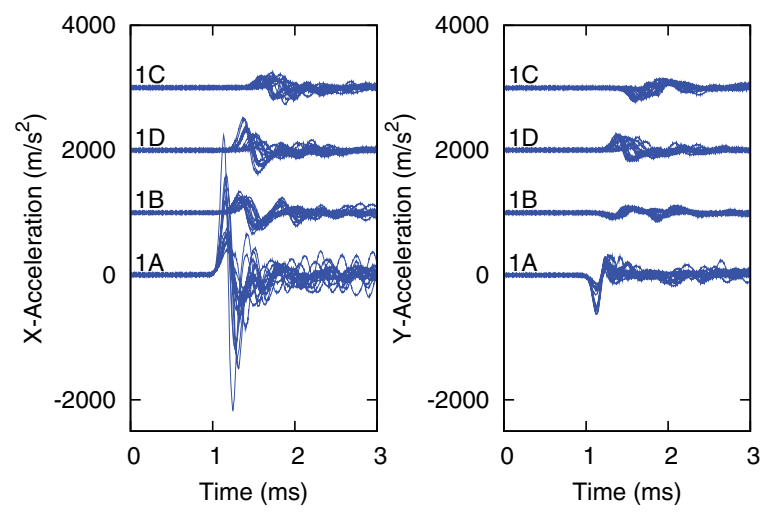

(b)
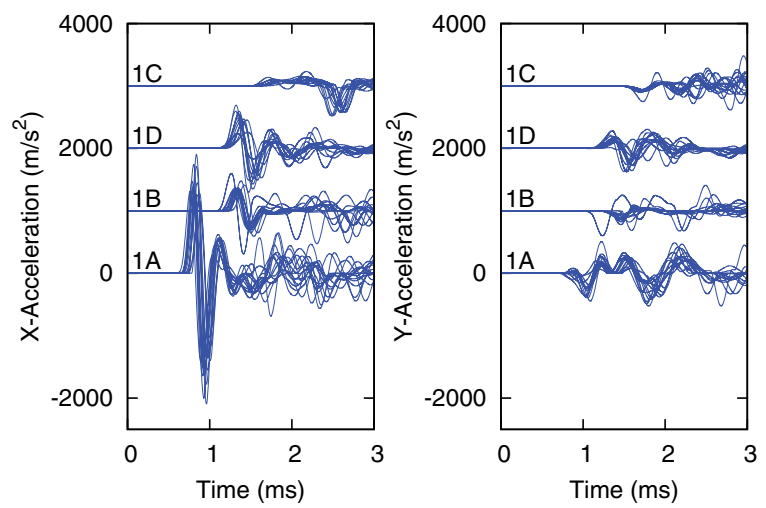

(d)

FIG. 7. (Color online) Experimental and numerical simulation results for the X-and Y-acceleration profiles for sensor configuration 1 (see Fig. 1) of the delrin ${ }^{\mathrm{s}}-\mathrm{ptfe}^{\mathrm{c}}$ granular crystal. (a) Simulation results for the ideal granular crystal. (b) Experimental results for a single packing (SR) impacted 15 times. (c) Numerical simulation results for 15 different realizations (MR), each impacted once. (d) Numerical simulation results for a single realization (SR), impacted 15 times. For visual clarity, the acceleration data at sensor locations $1 \mathrm{~B}, 1 \mathrm{D}$, and $1 \mathrm{C}$ are shifted by 1000,2000 , and $3000 \mathrm{~m} / \mathrm{s}^{2}$, respectively, from sensor location $1 \mathrm{~A}$. The zero time in the simulations denotes the moment of impact, while the zero time in experiments is arbitrary since the recorded data are based off the signal arrival time of sensor $1 \mathrm{~A}$. 


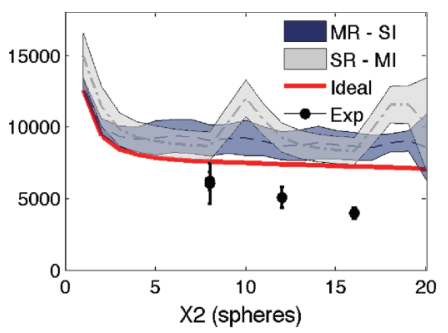

(a)

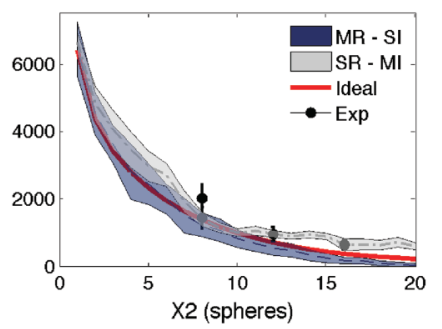

(b)

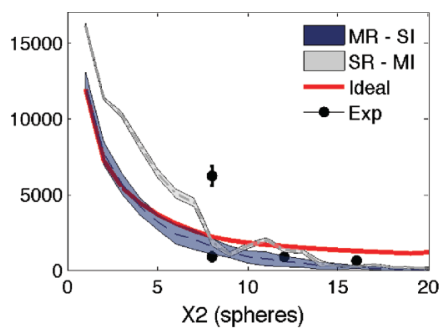

(c)

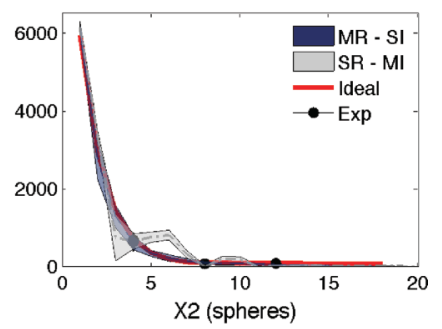

(d)

FIG. 8. (Color online) Peak acceleration $\left(\mathrm{m} / \mathrm{s}^{2}\right)$ in the wave front versus X2 (in units of spheres) for each sphere-cylinder material combination: (a) steel ${ }^{\mathrm{s}}-\mathrm{ptfe}^{\mathrm{c}}$, (b) delrin ${ }^{\mathrm{s}}-\mathrm{ptfe}^{\mathrm{c}}$ (c) steel $^{\mathrm{s}}$-steel ${ }^{\mathrm{c}}$, and (d) delrin ${ }^{\mathrm{s}}$-steel ${ }^{\mathrm{c}}$. The results from numerical simulations for the ideal granular crystal are shown by the solid (red) lines. The mean and standard deviation are represented by dashed lines and dark (blue) shaded regions for the MR-SI and by dot-dashed lines andlight (gray) shaded regions for the SR-MI. For each sensor location along X2, a set of black dots and error bars represent the mean and standard deviation for the single experimental realization with multiple impacts.

variability due to the presence of disorder in the system. We compare the numerical results obtained for a lattice with no disorder [Fig. 7(a)] and with experimental results [Fig. 7(b)] and results obtained numerically for the MR-SI [Fig. 7(c)] and SR-MI [Fig. 7(d)] cases. Similar to the experimental data, the numerical results show slight deviations from the ideal response for each realization. The individual particle responses corresponding to any one impact in experiments and numerical simulations are quantitatively different, but qualitatively similar. The variation in wave-front amplitude, initial pulse shape, and signal arrival times in numerical results are similar to those obtained experimentally for the delrin $^{\mathrm{s}}$-ptfe $^{\mathrm{c}}$ crystal (Fig. 7), as well as for the other three sphere-cylinder systems (not shown).

The individual acceleration profiles also show that variability in the tails of the signals is more pronounced than that in the initially arriving pulse. This effect is linked to the combination of small compressive forces and gaps present in the system as a result of disorder. The larger amplitude oscillations in the tails of the signals are also responsible for the more pronounced amplitude decay of the leading pulse, evident in the disordered systems.

In order to study the effects of disorder on the wave-front propagation along different radial directions within the crystal, we measure the wave-front peak acceleration distributions along the X2 (in-line with the impact) and X3 (along a $45^{\circ}$ angle to the impact) directions (refer to Fig. 1). We observe more variability between the amplitudes along $\mathrm{X} 1$ compared to those along X2 and X3. Sensors A, B, and C along X2 and X3 measure the peak acceleration along a line of particle contacts originating at the excitation, while sensors $\mathrm{A}, \mathrm{B}$, and $\mathrm{C}$ along $\mathrm{X} 1$ capture the relationship between the peak acceleration distributions along several radial directions from the impact.

In all cases, we observe good agreement between experiments and numerical results obtained including initial contact lattice disorder due to the particle tolerances. In experiments, additional sources of disorder could arise from imperfect orientations and surface textures of the confining walls. Also, minor variability in striker speed and impact locations can be present in the experiments. Variable striker speeds are expected to equally affect the measurements at all sensor locations, while off-center striker impacts are expected to result in asymmetries in the system response. We performed additional numerical simulations incorporating a uniform random distribution of particle diameters (not shown). A uniform probability distribution leads to more frequent and larger gaps in the initial contact lattice, that is, increased disorder. In numerical simulations, we observed significantly larger wave amplitude variability, corresponding to less distinguishable wave-front shapes between material combinations, for a uniform random distribution compared to the normal distribution of particle diameters. The level of disorder present in experiments was effectively captured by the numerical simulations incorporating the normal distribution

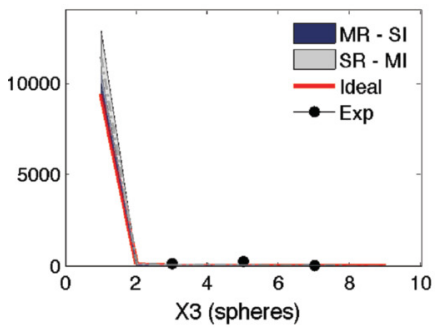

(a)

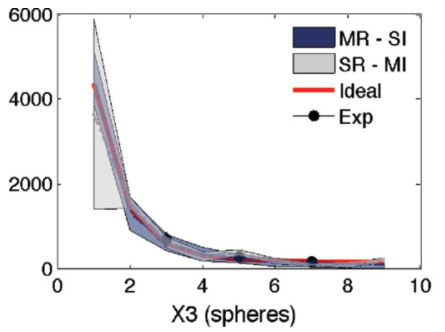

(b)

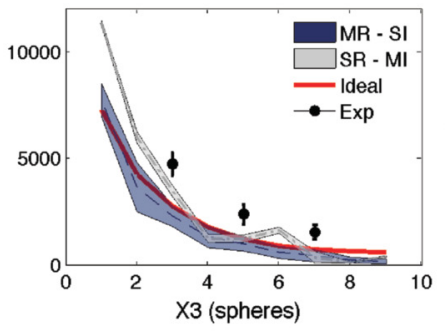

(c)

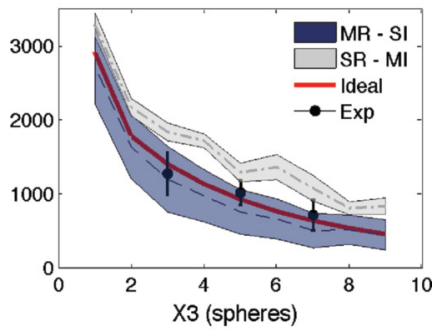

(d)

FIG. 9. (Color online) Peak acceleration $\left(\mathrm{m} / \mathrm{s}^{2}\right)$ in the wave front as a function of $\mathrm{X} 3$ (in units of spheres) for each sphere-cylinder material combination: (a) steel ${ }^{\mathrm{s}}-\mathrm{ptfe}^{\mathrm{c}}$, (b) delrin $-\mathrm{ptfe}^{\mathrm{c}}$ (c) steel $^{\mathrm{s}}-\mathrm{steel}^{\mathrm{c}}$, and (d) delrin ${ }^{\mathrm{s}}-\mathrm{steel}^{\mathrm{c}}$. The results from numerical simulations for the ideal granular crystal are shown by the solid (red) lines. The mean and standard deviation are represented by dashed lines and dark (blue) shaded regions for the MR-SI and by dot-dashed lines and light (gray) shaded regions for the SR-MI. For each sensor location along X2, a set of black dots and error bars represent the mean and standard deviation for the single experimental realization with multiple impacts. 
of particle diameters based on the specified precision ballbearing tolerances. Additionally, some discrepancies between trends observed in experiments and numerical simulations can be attributed to dissipation which are not accounted for in our numerical simulations. The presence of dissipation in experiments results in signal attenuation and time delays (Figs. 4, 6, 8, and 9).

The average particle responses from MR (both experimental and numerical) are consistent with the wave-front shapes predicted by numerical simulations of ideal granular crystals. For the steel ${ }^{\mathrm{s}}-\mathrm{ptfe}^{\mathrm{c}}$ and delrin ${ }^{\mathrm{s}}-\mathrm{ptfe}^{\mathrm{c}}$ granular crystals, the average particle responses from MR are in good agreement with the directional and triangular wave-front shapes, respectively [Figs. 4(a) and 4(b)]. Similarly, for the steel $^{\mathrm{s}}$-steel ${ }^{\mathrm{c}}$ and delrin ${ }^{\mathrm{s}}$-steel ${ }^{\mathrm{c}}$ systems, the average particle responses from MR are comparable with those predicted by the ideal system. However, the average response of the steel $^{\mathrm{S}}$-steel ${ }^{\mathrm{C}}$ weakly disordered systems produces a slightly more uniform distribution of peak acceleration in the wave front compared to the ideal system response [Fig. 4(c)]. Additionally, in both numerical simulations and experiments, the weakly disordered delrin $^{\mathrm{s}}$-steel ${ }^{\mathrm{c}}$ system results in the diverted impact energy, or squared wave-front shape to be more pronounced compared to that of the ideal crystal [Fig. 4(d)].

\section{CONCLUSIONS}

We investigated the stress wave propagation in a $2 \mathrm{D}$ granular crystal comprised of spheres arranged in a square lattice with cylindrical interstitial intruders. Experiments were compared with numerical simulations to capture the basic characteristics of the propagating wave fronts. We performed multiple experiments on the same crystal to capture the variability in the system response between successive impacts. We also assembled and tested several individual granular crystals to determine the effects of variability derived from differences in the initial contact lattices.
Numerical simulations were performed on nonrandom systems for a wide range of sphere-cylinder mass and stiffness ratios to characterize the properties of the traveling stress waves supported by this system. By systematically altering these two ratios, we observed a variety of propagating wave-front regimes, including directional, 2D pulsed, and 2D transitional propagation. Experiments were performed for systems in the directional and 2D transitional regime spanning a wide range of wave-front shapes.

Additional numerical simulations on weakly disordered systems incorporated particle diameter tolerances. We independently assessed the variability arising from differences in initial contact lattices and from particle rearrangements between successive impacts by performing tests for MR-SI and SR-MI. The agreements between the acceleration profiles in experiments and numerical simulations suggest that the main source of variability present in real systems derives from particle size imperfections which create a weakly disordered initial contact lattice.

In both experiments and numerical simulations, larger discrepancies existed between the average response of SR-MI and those of the ideal granular crystal, compared to the average response of the MR-SI. However, while discrepancies exist between individual tests, the average properties of the wave fronts for each of the four material configurations tested were consistent with those predicted by numerical simulations on ideal granular crystals, confirming the ability to systematically alter the properties in real 2D arrays. This work is a first step in designing granular crystals with predetermined wave propagation paths.

\section{ACKNOWLEDGMENTS}

This work was supported by an award from the Department of Energy Office of Science (DOE SCGF) and the Army Research Office MURI. Project No. W911NF0910436.
[1] K. Johnson, Contact Mechanics (The Press Syndicate of the University of Cambridge, Cambridge, 1987).

[2] V. Nesterenko, J. Appl. Mech. Tech. Phys. 24, 733 (1983).

[3] C. Coste, E. Falcon, and S. Fauve, Phys. Rev. E 56, 6104 (1997).

[4] V. F. Nesterenko, Dynamics of Heterogeneous Materials (Springer-Verlag, New York, 2001).

[5] C. Daraio, V. F. Nesterenko, E. B. Herbold, and S. Jin, Phys. Rev. E 72, 016603 (2005).

[6] A. Leonard, F. Fraternali, and C. Daraio, Exp. Mech. (2011), doi: 10.1007/s11340-011-9544-6.

[7] J. Geng, D. Howell, E. Longhi, R. P. Behringer, G. Reydellet, L. Vanel, E. Clement, and S. Luding, Phys. Rev. Lett. 87, 035506 (2001).

[8] N. W. Mueggenburg, H. M. Jaeger, and S. R. Nagel, Phys. Rev. E 66, 031304 (2002).

[9] L. Breton, P. Claudin, E. Clément, and J. Zucker, Europhys. Lett. 60, 813 (2002).
[10] J. Geng, G. Reydellet, E. Clément, and R. Behringer, Phys. D (Amsterdam, Neth.) 182, 274 (2003).

[11] T. Travers, M. Ammi, D. Bideau, A. Gervois, J. Messager, and J. Troadec, Europhys. Lett. 4, 329 (1997).

[12] C. Liu, S. Nagel, D. Schecter, S. Coppersmith, S. Majumdar, O. Narayan, and T. Witten, Science 269, 513 (1995).

[13] S. N. Coppersmith, C.-h. Liu, S. Majumdar, O. Narayan, and T. A. Witten, 4673 (1996).

[14] S. Bardenhagen and J. Brackbill, J. Appl. Phys. 83, 5732 (1998).

[15] K. Roessig, J. Foster, and S. Bardenhagen, Exp. Mech. 42, 329 (2002).

[16] E. T. Owens and K. E. Daniels, Europhys. Lett. 94, 54005 (2011).

[17] Y. Zhu, A. Shukla, and M. Sadd, J. Mech. Phys. Solids 44, 1283 (1996).

[18] Q. Tai and M. Sadd, Int. J. Numer. Anal. Methods Geomech. 21, 295 (1997).

[19] C. Daraio, D. Ngo, V. F. Nesterenko, and F. Fraternali, Phys. Rev. E 82, 036603 (2010). 
[20] D. Ngo, F. Fraternali, and C. Daraio, Phys. Rev. E 85, 036602 (2012).

[21] A. Awasthi, K. Smith, P. Geubelle, and J. Lambros, Mech. Mater. 54, 100 (2012).

[22] A. Leonard and C. Daraio, Phys. Rev. Lett. 108, 214301 (2012).

[23] J. Goddard, Proc. R. Soc. London A 430, 105 (1990).

[24] L. Vergara, Phys. Rev. Lett. 95, 108002 (2005).

[25] L. Ponson, N. Boechler, Y. M. Lai, M. A. Porter, P. G. Kevrekidis, and C. Daraio, Phys. Rev. E 82, 021301 (2010).

[26] M. Manjunath, A. P. Awasthi, and P. H. Geubelle, Phys. Rev. E 85, 031308 (2012).

[27] B. Gilles and C. Coste, Phys. Rev. Lett. 90, 174302 (2003).

[28] C. Coste and B. Gilles, Phys. Rev. E 77, 021302 (2008).

[29] O. Mouraille and S. Luding, Ultrasonics 48, 498 (2008).

[30] S. Sen and R. S. Sinkovits, Phys. Rev. E 54, 6857 (1996).

[31] M. Nishida, K. Tanaka, and T. Ishida, Shock Waves (Springer, Berlin, Heidelberg, 2009).

[32] E. Hascoët and H. Herrmann, Eur. Phys. J. B 14, 183 (2000).
[33] S. Job, F. Santibanez, F. Tapia, and F. Melo, Phys. Rev. E 80, 025602 (2009).

[34] M. Nishida and Y. Tanaka, Granular Matter 12, 357 (2010).

[35] R. Singh, A. Shukla, and H. Zervas, Int. J. Solids Struct. 32, 2523 (1995).

[36] www.mcmaster.com.

[37] A. Sokolow, E. Bittle, and S. Sen, Europhys. Lett. 77, 24002 (2007).

[38] S. Job, F. Melo, A. Sokolow, and S. Sen, Granular Matter 10, 13 (2007).

[39] M. Puttock and E. Thwaite, Elastic Compression of Spheres and Cylinders at Point and Line Contact (CSIRO, Melbourne, 1969).

[40] LAMMPS granular package, http://lammps.sandia.gov/doc/ pair_gran.html.

[41] S. Plimpton, J. Comput. Phys. 117, 1 (1995).

[42] K. W. Chase and W. H. Greenwood, Manuf. Rev. 1, 50 (1988). 